\title{
GeoBO: Python package for Multi-Objective Bayesian Optimisation and Joint Inversion in Geosciences
}

DOI: $10.21105 /$ joss. 02690

\section{Software \\ - Review ¿ \\ - Repository ut \\ - Archive ct}

Editor: Hugo Ledoux ¿

Reviewers:

- @npetra

- @sgkang

Submitted: 02 July 2020 Published: 28 January 2021

\section{License}

Authors of papers retain copyright and release the work under a Creative Commons Attribution 4.0 International License (CC BY 4.0).

\author{
Sebastian Haan ${ }^{1}$ \\ 1 Sydney Informatics Hub, The University of Sydney, Australia
}

\section{Summary}

A critical decision process in geophysical data collection is how to efficiently combine a variety of sensor types and where to collect new observations, in particular if measurements are very costly or resources are limited. This may include drill-core placements or the layout of a geophysical survey. Bayesian Optimisation (BO) solves this decision making problem by finding global optimal solutions given multiple, often distinct measurements and model uncertainties. One of the major challenges for applying $\mathrm{BO}$ in geoscience still lies in constructing a 3D model from sparse, typically 2D sensor data that can be computationally efficiently evaluated and that transforms the combined data and uncertainties from multiple sensor measurements coherently into a posterior function approximating the true model; this is also known as an inversion problem.

GeoBO is a Python package that solves both Multi-Objective Optimisation and Joint Inversion within one probabilistic framework: from prior selection and input, data fusion and inversion, to the final sensor optimisation and real world model output. Fig. 1 shows a graphical overview model of $\mathrm{GeoBO}$; the two main process steps are summarized in the following.

First, GeoBO jointly solves multi-linear forward models of 2D survey data (e.g., magnetics and gravity) to 3D-geophysical properties using Gaussian Process kernels (Melkumyan \& Ramos, 2009; Rasmussen \& Williams, 2006), without the requirement of prior geological knowledge. In addition, sparse drillhole measurements can be taken into account. One of the key features is to consider correlations between geophysical properties by solving simultaneously multiple forward models (Melkuyman \& Ramos, 2011; Reid et al., 2013). Thus, this approach provides a better constrained combined solution of multiple, distinct sensor types, rather than taking their individual solutions. Another practical advantage of this probabilistic approach is that predictions are described by posterior distributions for each location (voxel or cube cell), which quantify the uncertainty in the predictions and their credible intervals. Most other work in the field of joint inversion only report point estimates of the quantities of interest (GallardoDelgado et al., 2003; Moorkamp et al., 2011; Zeyen \& Pous, 1993).

While this inversion method is limited to linear forward models, the reconstructed posterior distribution of the geophysical properties can be further refined using site-specific knowledge or more complex inversion methods for sampling non-linear forward models, such as hIPPYlib (Villa et al., 2018), Obsidian (Scalzo et al., 2019), or GemPy (Varga et al., 2019).

In a second step, the $\mathrm{BO}$ in GeoBO allows the user to define objectives in an acquisition function (Brochu et al., 2010; Mockus, 1975; Sebastian Haan, 2020) which typically has to balance between a) exploration, i.e., querying points that maximise the information gain and minimize the uncertainty of a geophysical site model; b) exploitation, i.e., querying points that maximise the reward (e.g., concentrating search in the vicinity locations with high value such as minerals); and c) minimize the number of samples given a cost function for any new measurement (e.g., costly drill-cores). The reconstructed 3D model output of the joint 
inversion model is then used to query for the next most promising point based on the aquisition function, which guides the search for a user-defined optimum.

GeoBO allows applications to specify priors as additional options, such as a) the typical correlation lengthscale of the geological structure via GP hyperparameters, b) the gain/cost parameters in the BO acquisition function, and c) the correlation coefficients between different geophysical properties. This package includes forward models for gravity and magnetics surveys (Yaoguo \& Oldenburg, 1998), drillcores, and test-sets of geological models and data.

While it has been tested on solving the problem of allocating iteratively new expensive drillcore samples (Sebastian Haan, 2020), the same method can be applied to a wide range of optimisation and sensor fusion problems.

\section{Multi-Objective Bayesian Optimisation and Joint Inversion}

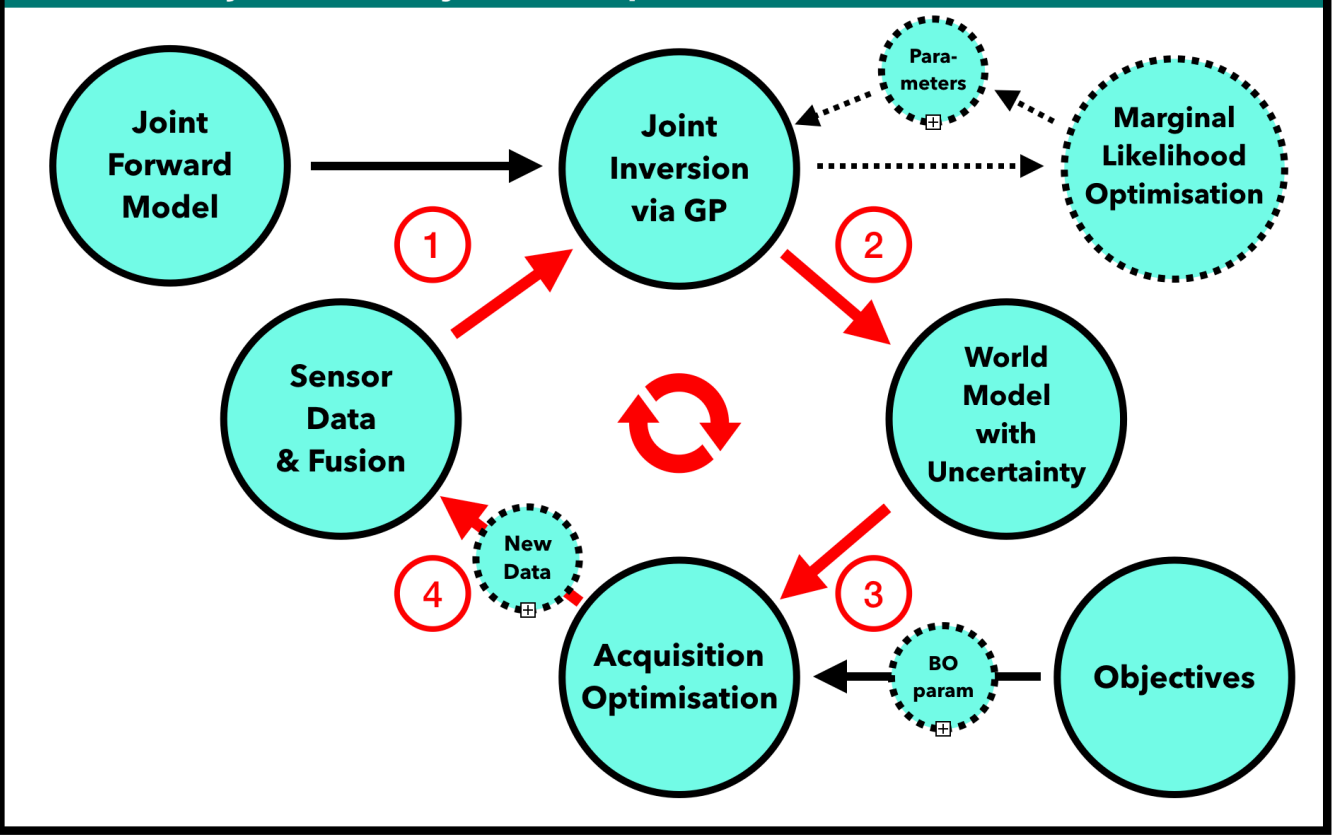

Figure 1: Overview of the probabilistic framework for multi-objective optimisation and joint inversion; the process stages are: 1) Joint inversion via Gaussian Processes based on multiple sensor data fusion and forward model, 2) Posterior model generation of 3D multi-geophysical properties. 3) Maximisation of acquisition function to allocate optimal new sensor location and type. 4) New acquired data is combined with existing data; process repeats until maximum number of iterations is achieved. For more details see Sebastian Haan (2020)

\section{Installation, Dependencies and Usage}

GeoBO can be installed using setuptools or pip and requires the following python packages: numpy, matplotlib, scikit_image, scipy, rasterio, pandas, pyvista, skimage, PyYAML (for details see requirements.txt). The installation can be tested by running the script with included synthetic data and default settings.

To use GeoBO, change the main settings such as filenames and parameters in settings.yaml (see description there), and run

cd geobo/

python main.py settings.yaml 
Core features of GeoBO:

- Joint probabilistic inversion tool by solving simultaneously multi-linear forward models (e.g. gravity, magnetics) using cross-variances between geophysical properties (crossvariance terms can be specified by use in settings.yaml)

- Computation of complete posterior distribution for all geophysical properties (described by their mean and variance value at each location/voxel)

- Generation of ranked proposal list for new most promising drillcores (optional: non-vertical drillcores) based on global optimisation of acquisition function (see settings.yaml)

- Templates for acquisition function to use in Bayesian Optimisation (see 'futility' functions in run_geobo.py)

- Flexible parameter settings (settings.yaml) for exploration-exploitation trade-off and inclusion of local 3D cost function in acquisition function (see function 'create_costcube' in run_geobo.py)

Other features are:

- Package includes geological survey/drillcore sample as well as synthetic data and functions for synthetic data generation

- Generation of 2D/3D visualisation plots of reconstructed cubes and survey data (see plot settings in settings.yaml)

- 3D Cube export in VTK format (for subsequent analysis, e.g., with ParaView)

- Options to include any pre-existing drillcore data (see settings.yaml for feature names as well as format of sample drilldata)

- Included linear forward models: density-to-gravity and magnetic susceptibility-tomagnetic field; custom linear forward models can be added (sensormodel.py)

- Library of Gaussian Process (GP) kernels including sparse GP kernels (kernels.py)

- Flexible settings for any cube geometry and resolution (cube length/width/height and voxel resolutions)

- Optional marginal GP likelihood for optimization of GP hyperparameters and inversion process

\section{Results and Output}

First, the joint inversion generates the reconstructed properties and their uncertainties as 3D cubes, such as density and magnetic susceptibility. Other 3D physical properties can be obtained by adding custom forward models (see section Custom Linear Forward Models in README). Secondly, Bayesian Optimisation uses these probabilistic inversion results to query new potential measurements based on a specific set of objectives as defined in an acquisition function, which guides the search for a user-defined optimum. Finally a list of all new measurement proposals ranked from maximum to minimum improvement is generated including a map of the most promising new measurement locations.

\section{Acknowledgements}

Sebastian Haan would like to acknowledge Dietmar Muller, Fabio Ramos, and Ben Mather for their very valuable contributions to this project. This research was supported by the Sydney Informatics Hub, a Core Research Facility of the University of Sydney. 


\section{References}

Brochu, E., Cora, V. M., \& De Freitas, N. (2010). A tutorial on Bayesian optimization of expensive cost functions, with application to active user modeling and hierarchical reinforcement learning. arXiv Preprint arXiv:1012.2599.

Gallardo-Delgado, L. A., Pérez-Flores, M. A., \& Gómez-Treviño, E. (2003). A versatile algorithm for joint 3D inversion of gravity and magnetic data. Geophysics, 68(3), 949-959. https://doi.org/10.1190/1.1581067

Melkumyan, A., \& Ramos, F. (2009). A sparse covariance function for exact Gaussian process inference in large datasets. International Joint Conference on Artificial Intelligence, 9, 1936-1942.

Melkuyman, A., \& Ramos, F. (2011). Multi-kernel Gaussian processes. International Joint Conference on Artificial Intelligence, 22, 1408.

Mockus, J. (1975). On Bayesian methods of optimization. Towards Global Optimization, $166-181$.

Moorkamp, M., Heincke, B., Jegen, M., Roberts, A. W., \& Hobbs, R. W. (2011). A framework for 3-D joint inversion of MT, gravity and seismic refraction data. Geophysical Journal International, 184(1), 477-493. https://doi.org/10.1111/j.1365-246X.2010.04856.X

Rasmussen, C. E., \& Williams, C. K. (2006). Gaussian process for machine learning. MIT press.

Reid, A., Simon Timothy, O., Bonilla, E. V., McCalman, L., Rawling, T., \& Ramos, F. (2013). Bayesian joint inversions for the exploration of earth resources. International Joint Conference on Artificial Intelligence, 2877-2884.

Scalzo, R., Kohn, D., Olierook, H., Houseman, G., Chandra, R., Girolami, M., \& Cripps, S. (2019). Efficiency and robustness in Monte Carlo sampling for 3-D geophysical inversions with Obsidian v0. 1.2: setting up for success. Geoscientific Model Development, 12(7), 2941-2960. https://doi.org/10.5194/gmd-12-2941-2019

Sebastian Haan, D. M., Fabio Ramos. (2020). Multiobjective Bayesian optimisation and joint inversion for active sensor fusion. Geophysics, 86(1), Pp.1-78. https://doi.org/10.1190/ geo2019-0460.1

Varga, M. de la, Schaaf, A., \& Wellmann, F. (2019). GemPy 1.0: open-source stochastic geological modeling and inversion. Geoscientific Model Development. https://doi.org/10. 5194/gmd-12-1-2019

Villa, U., Petra, N., \& Ghattas, O. (2018). hIPPYlib: An extensible software framework for large-scale inverse problems. Journal of Open Source Software, 3(30). https://doi.org/ $10.21105 /$ joss.00940

Yaoguo, L., \& Oldenburg, D. W. (1998). 3D-inversion of gravity data. Geophysics, 63(1), 109-119.

Zeyen, H., \& Pous, J. (1993). 3-D Joint Inversion of Magnetic and Gravimetric data with a priori Information. Geophysical Journal International, 112(2), 244-256. https://doi.org/ 10.1111/j.1365-246x.1993.tb01452.x 